\title{
GENE THERAPY: HOPES, HYPES, AND HURDLES
}

\author{
Inder M. Verma, Ph.D. \\ Molecular Biology and Virology Laboratory, \\ The Salk Institute, La Jolla, California, U.S.A.
}

There is universal agreement among scientists and laymen, skeptics and protagonists, that gene therapy is a novel form of molecular medicine that will have a major impact on human health in the next century. Rarely has a scientific idea or technology captured the imagination of so many, and therein lie the unrealistic expectations. What then is gene therapy? It is the introduction of a gene into a somatic tissue so that its product can alleviate the defect caused by the loss or malfunctioning of a vital gene product. Minimum requirements for successful gene therapy will entail: (i) no undesirable side effects, and (ii) sustained production of therapeutic gene product. The scope of gene therapy has expanded with the possibility of providing treatment for acquired maladies like cancer, AIDS, and neurological disorders like Parkinsons and Alzheimers disease. While the definitions and goals of gene therapy have been sharply focused, the actual execution of the task has not kept pace due to many unanticipated roadblocks.

\section{HOPES}

To transfer the therapeutic gene product into somatic tissues, a number of vector systems have been developed. The broad category of physical or chemical delivery methods including DNA transfections, direct DNA injection, receptorligand complexes, electroporation, etc. are beneficial if the expression of the therapeutic gene product is required for a transient period. The direct injection approach holds exciting prospects of developing "DNA vaccines". Biological vectors are the system of choice to achieve therapeutic levels of the desired gene product, such as production of 1 to $5 \mu \mathrm{g}$ of factor IX (hemophilia B) protein per $\mathrm{ml}$ of plasma. The nature of the bio- logical vector is dictated by the choice of ex vivo or in vivo gene delivery, and transient or sustained expression. Retroviral vectors are by far the most extensively employed because of their ability to integrate in the host chromosome and allow sustained expression. Unfortunately, integration by retroviral vectors requires dividing cells which precludes their use for in vivo gene delivery in somatic tissues like brain, liver, muscle, etc. Adenovirus based vectors have two major advantages: (i) high titer recombinant viruses (about $10^{11} / \mathrm{ml}$ ) can be easily generated; (ii) postmitotic cells can be efficiently transduced because adenoviral DNA exists as an episome in the nucleus. Thus recombinant adenoviruses can be used for in vivo gene delivery but expression of the foreign gene is transient and requires reinfection. More recently adeno-associated viral (AAV) vectors have become a favorite because they can integrate in non-dividing cells. There is, however, substantial controversy as to whether AAV integrates in non-dividing cells or exists as an episome in quiescent cells and integrates upon cell replication. The herpes virus based vectors are still in their early development state and need considerable further study before being considered for gene therapy.

A number of somatic tissues have been explored for transduction of foreign genes, prominent among them are bone marrow, fibroblasts, muscle, skin, liver, brain, etc. The precise choice of the tissue is dictated by the nature of the malady that needs to be corrected. The ex vivo approach can be contemplated, only if reimplantation of the somatic tissue is feasible. Another important handicap of an ex vivo approach is that the results obtained from experimental model systems may not be applicable to humans. Our own experience in this regard is instructive in that while mouse myoblasts fuse efficiently to 
form myotubes, canine myoblasts fuse too inefficiently to provide effective therapy for hemophilic dogs.

Despite many difficulties, it is fair to say that a wide variety of vectors and somatic tissues have been identified which have the potential to fulfill the promise of gene therapy.

\section{HYPES}

When gene therapy was first contemplated, there was healthy skepticism in the scientific community. Initial success in generating helperfree retroviral vectors, high titer adenoviral vectors and direct DNA injection, followed by efficient transduction of therapeutic genes in a variety of somatic tissues, opened the floodgates of promise by gene therapy. The rush to undertake clinical trials further fueled the heightened expectations. Every time a new gene is identified and molecularly cloned, there are immediate implications of gene therapy. Mere approval of the recombinant advisory committee (RAC) to undertake phase one of tumor marking trials are not tantamount to successful gene therapy. Fortunately, a vast majority of the scientific community is aware of both the technical and practical limitations of current approaches to gene therapy.

\section{HURDLES}

It was only a decade ago that high-efficiency and replication-defective retroviral vectors containing therapeutic genes were generated. The adenoviral and adeno-associated viral vectors are even more recent and thus it should not come as a surprise that there are plenty of hurdles in using these vectors towards successful gene therapy. Problems abound with the generation of high-titer (more than $10^{6}$ to $10^{7}$ virus particles/ $\mathrm{ml}$ ) recombinant retroviruses. Recent use of VSVG protein to pseudotype retroviruses offers a new avenue of increasing viral titers. Inability of retroviral vectors to infect post-mitotic cells remains a serious handicap for in vivo gene delivery, but again hope is on the horizon in the form of lentiviruses capable of infecting non-dividing cells. Another unexpected obstacle encountered with retroviral vectors is the loss of expression of the transgene following in vivo implantation. Choice of appropriate enhancer-promoters can overcome some of these problems, but nevertheless they require further exploration. Currently available adenoviral vectors are not totally replication-defective and when used at high-titers can lead to the elimination of infected cells by cytotoxic T lymphocytes. Novel adenoviral vectors further debilitated to allow any viral replication are being generated which may overcome this problem. The other major problem of adenoviral vectors is the humoral response to input viral antigens. Since adenoviruses remain in an episomal state, sustained expression requires reinfection which can be compromised by neutralizing antibodies to viral proteins. The extent of the humoral response is dictated by the titers of the infecting virus and may be overcome by using different serotypes of adenoviral vectors. Currently the available adeno-associated viral vectors do not generate high-titer recombinant viruses and the extent of integration in nondividing cells is still somewhat uncertain. It is, however, clear that, unlike wild type AAV, recombinant AAV do not integrate in a site specific manner.

Despite the lack of ideal vectors, there has been tremendous progress in the variety of approaches being developed for gene delivery. Over 100 clinical trials, the majority of them on cancer patients, are currently in progress. Initial results on the introduction of ADA gene in two young children are cautiously optimistic. What is needed now is more basic understanding of the biology of the variety of viruses being used as vectors. Development of alternative delivery systems also needs to be vigorously explored. In addition, the cell biology of the somatic tissues being considered for transducing genes needs to be further investigated (in particular, the identification and manipulation of the stem cells). Though successful gene therapy as routine medical practice is still distant, substantial current efforts of basic scientists and clinicians are likely to hasten the transfer of bench science to the bedside. 